27. Borowitz MJ, Craig FE, Digiuseppe JA, et al. Guidelines for the diagnosis and monitoring of paroxysmal nocturnal hemoglobinuria and related disorders by flow cytometry. Cytometry B Clin Cytom. 2010;78(4):211-30.

28. Peacock-Young B, Macrae FL, Newton DJ, Hill A, Ariens RAS. The prothrombotic state in paroxysmal nocturnal hemoglobinuria: a multifaceted source. Haematologica. 2018;103(1):9-17.
29. Wiedmer T, Hall SE, Ortel TL, Kane WH, Rosse WF, Sims PJ. Complement-induced vesiculation and exposure of membrane prothrombinase sites in platelets of paroxysmal nocturnal hemoglobinuria. Blood. 1993;82(4):1192-96.

30. Young NS, Meyers G, Schrezenmeier H, Hillmen P, Hill A. The management of paroxysmal nocturnal hemoglobinuria: recent advances in diagnosis and treatment and new hope for patients. Semin Hematol. 2009;46(1 Suppl 1):S1-S16.
31. Nishimura J, Kanakura Y, Ware RE, et al. Clinical course and flow cytometric analysis of paroxysmal nocturnal hemoglobinuria in the United States and Japan. Medicine (Baltimore). 2004;83(3):193-207.

32. Ricklin D, Mastellos DC, Reis ES, Lambris JD. The renaissance of complement therapeutics. Nat Rev Nephrol. 2018;14 (1):26-47.

\title{
Paroxysmal nocturnal hemoglobinuria: patient journey and burden of disease
}

\author{
Meryem Bektas, PhD, MBA; Catherine Copley-Merriman, MS, MBA; Shahnaz Khan, MPH; \\ Sujata P Sarda, BPharm, PhD; and Jamile M Shammo, MD, FASCP, FACP
}

\section{SUMMARY}

Patients with paroxysmal nocturnal hemoglobinuria $(\mathrm{PNH})$ often experience a lengthy path to diagnosis. Fewer than $40 \%$ of patients with $\mathrm{PNH}$ receive a diagnosis within 12 months of symptom onset, and $24 \%$ of all PNH diagnoses can take 5 years or longer. Diagnostic delay is a source of distress and can affect emotional well-being for patients with $\mathrm{PNH}$. In $\mathrm{PNH}$ disease management, patients and care providers focus on risk of organ failure and mortality related to disease progression; nonetheless, patients' healthrelated quality of life (HRQOL) is largely affected by extensive treatment requirements and nonfatal complications of disease, such as fatigue. In particular, thrombosis is associated with significant impairments in physical and social functioning and global health status and significant fatigue. Among patients with anemia who are transfusion dependent, the burden of transfusion is considerable. Transfusion dependence has a negative effect on HRQOL; is associated with risks and complications, including iron overload; and results in lost productivity due to travel times to and time spent at infusion centers.

\section{Author affiliations}

Meryem Bektas, PhD, MBA; Catherine Copley-Merriman, MS, MBA; and Shahnaz Khan, MPH, Market Access and Outcomes Strategy, RTI Health Solutions, Research Triangle, NC. Sujata P Sarda, BPharm, PhD, Global Health Economics and Outcomes Research, Apellis Pharmaceuticals, Waltham, MA, and Jamile M Shammo, MD, FASCP,

FACP, Department of Internal Medicine, Rush University Medical Center, Chicago, IL.

\section{AUTHOR CORRESPONDENCE: \\ Meryem Bektas, 919.541.6776; \\ mbektas@rti.org}




\section{The Patient Journey}

\section{PATH TO DIAGNOSIS}

Paroxysmal nocturnal hemoglobinuria $(\mathrm{PNH})$ is multisystemic with nonspecific symptoms and clinical manifestations due to intravascular hemolysis, thrombosis, and bone marrow failure. ${ }^{1}$ As a result of the multifactorial symptoms of $\mathrm{PNH}$, many patients experience a lengthy and complex path to diagnosis. ${ }^{2}$ According to an online survey of 163 patients with $\mathrm{PNH}$, on average, it takes close to 2 years and often multiple providers to correctly diagnose $\mathrm{PNH} .^{3,4}$ Fewer than $40 \%$ of patients with $\mathrm{PNH}$ receive their diagnosis within 12 months of symptom onset, and 24\% of all PNH diagnoses can take 5 years or longer. ${ }^{4}$ Moreover, $79 \%$ of patients consult more than 1 physician before receiving a diagnosis, and of these patients, approximately $38 \%$ see 5 or more different physicians across different specialties, such as primary care physicians, hematologists, obstetrician-gynecologists, urologists, mental health specialists, pulmonologists, and neurologists. ${ }^{4}$

The length of time to diagnosis can be a source of distress and affect the patient's emotional well-being, in addition to carrying clinical implications. Patients with PNH are at risk for complications of the disease before diagnosis: It is estimated that $40 \%$ of patients have experienced a thromboembolic event before diagnosis. ${ }^{5}$ In addition, the length to and the uncertainties surrounding a diagnosis can be psychologically distressing for patients and their families. $^{3}$ The survey of 163 patients with $\mathrm{PNH}$ found that some patients described their journey to a diagnosis as "periods of hopelessness and anxiety" that had a long-lasting effect on their lives. ${ }^{3}$ Because $\mathrm{PNH}$ can present with changing symptoms, care episodes appeared disconnected for many patients, and some felt isolated, anxious, and dismissed by medical personnel. ${ }^{3}$ Despite the potential severity, most patients felt relief once the diagnosis of $\mathrm{PNH}$ was reached; subsequently, patients felt fear, since few were aware of this type of disease. Although $54 \%$ of patients consulted a primary care physician, approximately $15 \%$ went to the emergency department to receive care. A minority of patients saw hematologists or obstetrician-gynecologists for an initial evaluation (11\% and 7\%, respectively). Only one third of these patients seeking help for their symptoms were later referred to a hematologist. ${ }^{3}$

Patients who present with a Coombs-negative hemolytic anemia, aplastic anemia, refractory anemia, and unexplained thrombosis, especially in atypical locations (cerebral, dermal, and intra-abdominal vein thrombosis, and Budd-Chiari syndrome) co-occurring with cytopenia or hemolysis, are generally screened for $\mathrm{PNH}^{6}{ }^{6}$ If $\mathrm{PNH}$ is suspected, the physician may order different blood tests. ${ }^{6}$ Flow cytometric evaluation of the peripheral blood to assess for glycosylphosphatidylinositol (GPI)-anchored protein in at least 2 lineages (i.e., red and white blood cells) is the gold standard for diagnosis, as it is the most sensitive and reliable diagnostic test that confirms the presence of a PNH clone. ${ }^{7}$ Flow cytometry allows not only the detection of GPI-deficient cells but also the ability to quantify the proportion of the mutant cells and the specific population of blood cells most affected by the mutation.

\section{Burden of Disease}

\section{HEALTH-RELATED QUALITY-OF-LIFE BURDEN}

In $\mathrm{PNH}$ disease management, physicians largely focus on the risks of organ failure and mortality related to disease progression. Although these areas are of interest in the treatment of the disease, patients' health-related quality of life (HRQOL) is largely affected by nonfatal manifestations of disease progression, such as fatigue, and extensive treatment requirements. ${ }^{8}$ These complications limit a patient's ability to complete normal daily activities. ${ }^{9}$ There is currently a lack of literature using disease-specific measurement tools to adequately document the patient experience in PNH. Studies have relied on measures such as the European Organization for Research and Treatment of Cancer Quality-of-Life Questionnaire (EORTC QLQ-C30) and the Functional Assessment of Chronic Illness Therapy-Fatigue subscale (FACIT-Fatigue), which are used in evaluations of HRQOL among patients with cancer, to collect data from the PNH population. ${ }^{9,10}$

However, development efforts for $\mathrm{PNH}-$ specific patientreported outcome (PRO) measures are underway. The $\mathrm{PNH}$ Symptom Questionnaire (PNH-SQ), which was developed in accordance with U.S. Food and Drug Administration guidance for PRO measures, has demonstrated content validity in patients with $\mathrm{PNH}$ and will undergo psychometric evaluation using clinical trial data. ${ }^{11}$ In addition, an aplastic anemia/PNH-specific quality-of-life instrument has been developed, iteratively refined, and is currently undergoing psychometric evaluation, with plans to include the tool in the assortment of questionnaires used with the International PNH Registry. ${ }^{10,12}$

International PNH Registry. The International PNH Registry was initiated to evaluate disease burden, progression, and clinical outcomes for patients with $\mathrm{PNH} .{ }^{13,14}$ As of July 2017, the registry had enrolled 4,439 patients worldwide. ${ }^{14}$ An analysis of data from the registry was conducted to evaluate the participants' HRQOL using the FACIT-Fatigue and EORTC QLQ-C30, version 3.0. ${ }^{14}$ FACIT-Fatigue scores (among 1,894 patients with available data) indicated a clinically meaningful level of fatigue (median [Q1, Q3] scores of 
$34.0[27.0,40.0])$. FACIT-Fatigue scores range from 0 to 52 , with higher scores indicating less fatigue. EORTC QLQ-C30 Global Health Status (GHS)/Quality of Life (QOL) scores (among 1,888 patients with available data) indicated impaired HRQOL (median [Q1, Q3] scores of 58.3 [41.7, 75.0]). EORTC QLQ-C30 scores range from 0 to 100, with higher scores indicating better HRQOL. Patients enrolled in the registry experienced considerable disease-related fatigue and impairment in overall HRQOL relative to normative reference mean (SD) scores for the general adult population (FACIT-Fatigue: 43.6 [9.4]; EORTC QLQ-C30 Global Health/ QOL: $75.5[19.8])^{14,15}$

For patients with $\mathrm{PNH}$, thrombosis in particular is associated with significant impairments in physical and social functioning and global health status and significantly greater fatigue..$^{13}$ In an analysis of the first 1,610 patients enrolled in the registry worldwide, patient-reported EORTC QLQ-C30 and FACIT-Fatigue assessments indicated that, when compared with patients without a history of thrombosis, patients with a past thrombosis event had significantly lower GHS/QOL, physical functioning, and social functioning and significantly worse fatigue. In addition, patients reporting abdominal pain, chest pain, confusion, dysphagia, dyspnea, erectile dysfunction, fatigue, headache, hemoglobinuria, or scleral icterus in the 6 months before the study had statistically significantly worse HRQOL scores for all EORTC domains compared with patients who had not experienced each of those symptoms. ${ }^{13}$ Mean EORTC QLQC30 scores for the GHS of 63.7 and 57.5 in patients with PNH without and with a history of thrombosis, respectively, indicate that PNH has a clinically meaningful effect on HRQOL in all patients (at a 5-point threshold, relative to a reference score of 71.2 for the general population). ${ }^{13}$ In addition, mean FACIT-Fatigue scores of 35.9 and 33.4 in patients without and with a history of thrombosis, respectively, indicate a clinically meaningful greater level of fatigue (at a 3 -point threshold, relative to reference scores of 43.6 in the general population and 40.0 in patients with cancer without anemia). ${ }^{13}$

Surveillance Registry in Japan. As part of postmarketing surveillance, 491 patients with PNH who were treated with eculizumab after approval were registered in a postmarketing surveillance data registry in Japan as of March 2017. Among the patients enrolled between June 2010 and March 15, 2017, patients with available QOL data at baseline and at 1 year after eculizumab treatment were chosen for analysis $(n=54)$. Quality of life was assessed using the FACIT-Fatigue version 4 in Japanese and EORTC QLQ-C30 version 3 in Japanese. ${ }^{16}$
Overall, results showed that, after administration of eculizumab over 1 year, most QOL domains improved in comparison with baseline. In particular, significant improvement of EORTC QLQ-C30 scores was observed in fatigue, dyspnea, physical function, and GHS. Decreases in lactic dehydrogenase (LDH) and increases in hemoglobin showed strong correlations with QOL improvement. QOL improvement was independent of patients' baseline characteristics of co-occurrence of bone marrow failure or the degree of LDH level. ${ }^{16}$

FACIT-Fatigue and EORTC QLQ-C30 GHS and functioning scores indicated clinical and statistical improvements after 1 year of eculizumab treatment. ${ }^{16}$ For EORTC QLQC30 symptom scales, most showed clinical and statistical improvement at 1 year, particularly fatigue (mean [SD] scores of 61.2 [32.5] at baseline and 34.7 [25.3] at 1 year; $P<0.01$ ) and dyspnea (61.6 [37.2] vs. 37.1 [34.4], respectively; $P<0.01) .{ }^{16}$ Despite improvements with treatment, however, patients in this study treated with eculizumab for 1 year experienced continued disease-related fatigue and impairment in overall QOL relative to normative reference scores for the general adult population. ${ }^{14-17}$

Genetic variants in C5 do exist, and poor response to treatment has been reported among Japanese patients with PNH who received eculizumab. ${ }^{18}$ The prevalence of this mutation among the patients with PNH (3.2\%) was similar to that among healthy Japanese persons (3.5\%). This polymorphism was also identified in a Han Chinese population. A patient in Argentina of Asian ancestry who had a poor response had a very similar mutation..$^{18}$

\section{BURDEN OF CHRONIC ANEMIA, FATIGUE, AND TRANSFUSION}

Chronic anemia, fatigue, and the need for transfusion are common outcomes for patients with PNH. While evidence of the clinical and HRQOL burden in patients with PNH is limited, evidence from other disease areas, including oncology, suggests that the burden of chronic anemia, fatigue, and the need for transfusion are considerable.

Chronic Anemia. Anemia is a symptom of an underlying disease of various etiologies. Generally, anemia develops when there is an imbalance between production and/or release of red blood cells (RBCs) by the bone marrow and the loss of RBCs in the circulation. ${ }^{19}$ The imbalance caused on either side can stem from multiple causes, such as malnutrition or bone marrow failure leading to decreased production or release of RBCs and inherited or acquired hemolytic anemia leading to increased loss of RBCs. ${ }^{19}$

Chronic anemia is detrimental to organ function, as it results in a decreased oxygen-carrying capacity of the blood. During the short term, the body is able to counteract 
with an increase in heart rate and respiratory rate; left untreated, severe anemia can cause multi-organ failure. This can include high output heart failure, enlarged heart, myocardial infarction, angina, arrhythmias, cognitive impairment, and renal failure, among other conditions. ${ }^{19-21}$ In pregnant women, untreated anemia can cause premature birth and low birth weight and has been shown to be a costly complication that exacerbates ongoing hemolysis in $\mathrm{PNH}^{19,22,23}$

In patients with myelodysplastic syndrome (MDS), which is another bone marrow disorder that can be associated with $\mathrm{PNH}$, anemia is a major cause of morbidity, and patients with MDS who have anemia have increased mortality rates. ${ }^{24}$ Mortality is mainly mediated through increased occurrences of cardiovascular disease (CVD). ${ }^{24}$ Specifically, anemia in patients with MDS may lead to cardiac remodeling, cardiac enlargement, and left ventricular hypertrophy and may intensify ischemia and angina. In fact, up to half of all mortality in patients with MDS is related to CVD.

Fatigue. Anemia and low hemoglobin concentrations have been strongly associated with fatigue in patients with cancer. ${ }^{25}$ Fatigue significantly affects overall well-being and interferes with daily activity and work productivity. ${ }^{25}$ An analysis of data obtained from 5 randomized clinical trials demonstrated a significant and positive relationship between increasing hemoglobin level and reductions in fatigue. In particular, patients with a hemoglobin improvement of $\geq 2 \mathrm{~g} / \mathrm{dL}$ reported significantly greater increases in FACIT-Fatigue subscale scores than patients who did not have the same hemoglobin response. ${ }^{25}$ This translated into moderate improvements in physical, emotional, and functional well-being; large improvements in energy and activity levels; perceived overall health; and reduced need for assistance and, therefore, reduced burden for caregivers. ${ }^{25,26}$

An international, prospective, cohort observational study of high-risk (progression to acute myeloid leukemia) patients with MDS revealed that a higher degree of anemia $(\mathrm{P}<0.001)$ was associated with greater fatigue. ${ }^{27}$ Patients who reported a higher level of fatigue had an overall greater symptom burden, such as moderate to severe appetite loss and dyspnea, than patients who reported a lower level of fatigue. Anemia has many symptoms, including shortness of breath, headache, and chest pain, but patients perceived fatigue as having a greater negative effect on their daily lives than many other cancers or treatment-related complications, such as pain and nausea, with important emotional and mental repercussions, such as lack of self-motivation, sadness, frustration, and mental exhaustion. ${ }^{28}$ Fatigue is felt as diminished energy level and slowness that interferes with the patient's normal daily routine. Mentally and psychologically, fatigue is shown to affect typical cognitive function, including concentration and memory. ${ }^{28}$ Fatigue can also interfere with willingness to continue and adhere to cancer therapy and thus limit the amount of treatment that a patient tolerates, ultimately affecting disease outcomes..$^{29,30}$

Transfusion. Anemia is often treated with RBC transfusions, and chronic anemia in patients with PNH may result in transfusion dependence. ${ }^{31}$ Iron overload is a consequence of chronic transfusions and is associated with an elevated risk of morbidity and mortality in patients with MDS. ${ }^{22}$ Chronic transfusion may lead to refractory anemia and development of iron overload because the body cannot effectively excrete excess iron. ${ }^{32}$ Iron is, therefore, deposited in parenchymal tissues and in reticuloendothelial cells, and without a chelating therapy, iron overload can cause progressive damage to the liver, heart, endocrine system, brain, and joints. ${ }^{32,33}$ Hepatomegaly, liver dysfunction, heart failure, skin pigmentation, hypogonadism, diabetes mellitus, or arthropathy may occur in patients experiencing iron overload. ${ }^{32}$ A retrospective evaluation of 13 transfusion-dependent patients (>2 units per month) with acquired chronic refractory or inherited anemia who were reliant on transfusions for more than a year showed that 10 patients had abnormal liver function and 4 patients were diagnosed with heart failure. ${ }^{32}$ Serum ferritin levels increased from 1,830 to $5,740 \mathrm{ng} / \mathrm{mL}$ in all patients, and skin pigmentation, liver dysfunction, and endocrine dysfunction were observed in 9 patients with serum ferritin $>3,500 \mathrm{ng} / \mathrm{mL}, 8$ of whom died.

Transfusion-dependent patients may progress to secondary iron overload with organ impairment, which may be fatal in those who are heavily iron overloaded..$^{32} \mathrm{~A}$ study of transfusion-dependent Japanese patients with MDS, aplastic anemia, and other conditions concluded that mortality is higher in heavily iron-overloaded patients, caused primarily by liver and cardiac dysfunction. ${ }^{33}$ This study followed 292 patients who received a mean of 61.5 units of RBCs in a year. There were 75 deaths, of which $24 \%$ and $6.7 \%$ from these studies were reported as cardiac and liver failure, respectively, which were attributed to iron overload. ${ }^{33}$

Transfusion dependence has a negative effect on a patient's HRQOL and also requires substantial resources, including hospital admissions. ${ }^{22}$ A systematic literature review of the burden associated with chronic RBC transfusions in patients with MDS showed that transfusion-related risks and complications can include allergic or anaphylactic transfusion reactions, infections, development of antibodies to RBCs or other blood components, and skin rashes. Regardless of MDS risk score, patients with MDS who required transfusion had reduced overall survival relative to patients with MDS who did not require transfusions. ${ }^{22}$ Although transfusions can increase hemoglobin level acutely, patients can still experience long periods of 
suboptimal hemoglobin level, which can cause fatigue in addition to the physiologic effect of iron overload and cardiac events increasing risk of mortality. ${ }^{22}$

Transfusion is also associated with time burden and lost productivity due to travel to infusion centers..$^{34,35}$ To assess the burden of transfusion on patients, in terms of time spent, a retrospective chart review at multiple outpatient centers in the United States of patients with cancer receiving transfusion was conducted. ${ }^{34}$ The mean elapsed time between pretransfusion and posttransfusion vital sign assessment was 4.2 hours (95\% $\mathrm{CI}=3.64-4.81$ ), including 3.6 hours (95\% CI $=3.0-4.1$ ), on average, for patients to receive the actual $\mathrm{RBC}$ transfusion treatment. Patients had also an average one-way travel time of 30.0 minutes $(95 \% \mathrm{CI}=25.9-34.3){ }^{34}$

In addition, a pilot study of 120 patient records from a private oncology practice or local institutional transfusion center in the United States found that the average patient visit to an outpatient facility ranged from 3.55 hours for 1 packed red blood cell (PRBC) unit to 6.85 hours for 3 PRBC units; $90 \%$ of the patients at the facility received 2 PRBC units. ${ }^{35}$ In addition to costs associated with pretransfusion testing, transfusion administration, and transfusion-related complications, a number of U.S. national organizations have ongoing initiatives to reduce the use of transfusions. ${ }^{36,37}$ Programs have found reduction of 2 units of blood on a regular basis reduced mortality by $18 \%$ and resulted in significant cost savings (e.g., \$600,000) to large U.S. health systems. ${ }^{38}$

Taking all factors into account, time burden associated with transfusion may be substantial, which is often in addition to the time burden of chemotherapy treatments considerably affecting the patient's QOL. ${ }^{34,39}$

\section{CAREGIVER BURDEN}

Patients with a low hemoglobin level $(\leq 12 \mathrm{~g} / \mathrm{dL})$ may not be able to work at all, affecting primary caregivers. Caregivers may experience significant impact on their occupational productivity because of the need to reduce their work hours and days. ${ }^{28}$

\section{ECONOMIC BURDEN}

Productivity costs are particularly important for PNH because of the substantial time commitments required from patients and their caregivers for the intravenous administration of treatments (either eculizumab or ravulizumab) at infusion clinics. Levy et al. $(2019)^{40}$ compared the lost productivity for patients in the United States with PNH managed with eculizumab (administered every 2 weeks ${ }^{41}$ ) and ravulizumab (administered every 8 weeks ${ }^{42}$ ) delivered in an infusion clinic and at home.

A set of assumptions were generated using the literature and expert opinion to establish the total time needed for treatment, including administration, recovery, and travel times for each treatment regimen. The total duration was multiplied by an hourly wage of $\$ 20$ to estimate lost productivity. Results showed that treating 100 patients with $\mathrm{PNH}$ in a clinic for 2 years with eculizumab is estimated to result in a total duration of 25,920 hours in travel, administration, and recovery, yielding $\$ 518,400$ in lost productivity. The less frequent dosing of ravulizumab resulted in $\$ 184,800$ lost productivity (reduction of 64\%). Relative to clinical settings, home treatment reduced lost productivity by $\$ 320,000$ (38\%) for eculizumab and $\$ 154,000$ (70\%) for ravulizumab.

\section{Conclusions}

Fewer than $40 \%$ of patients with $\mathrm{PNH}$ received their diagnoses within
12 months of symptom onset, and a quarter of all PNH diagnoses can take 5 years or longer. Diagnostic delay can be a source of distress and affect patients' emotional well-being. $\mathrm{PNH}$ is characterized by intravascular and extravascular hemolysis, leading to severe anemia and other debilitating symptoms. If untreated, anemia can cause multi-organ failure, including heart failure, angina, arrhythmias, cognitive impairment, and renal failure. RBC transfusion is time consuming for patients and may lead to iron overload, potentially causing progressive damage to the liver, heart, and endocrine system. Many patients (80.9\%) report experiencing fatigue, which may result in loss of independence, decreased physical activity, and functional decline, if severe or left untreated..$^{14,16,43}$

\section{DISCLOSURES}

This research was developed under a research contract between RTI Health Solutions and Apellis Pharmaceuticals and was funded by Apellis Pharmaceuticals. Bektas, Copley-Merriman, and Khan are employees of RTI Health Solutions. Sarda is an employee of Apellis Pharmaceuticals. Shammo consults for Apellis Pharmaceuticals.

\section{ACKNOWLEDGMENTS}

Kate Lothman of RTI Health Solutions provided medical writing services, which were funded by Apellis Pharmaceuticals.

\section{REFERENCES}

1. Sahin F, Akay OM, Ayer M, et al. PESG PNH diagnosis, follow-up and treatment guidelines. Am J Blood Res. 2016;6(2):19-27.

2. Röth A, Maciejewski J, Nishimura JI, Jain D, Weitz JI. Screening and diagnostic clinical algorithm for paroxysmal nocturnal hemoglobinuria: expert consensus. Eur J Haematol. 2018;101(1):3-11. 
3. Mitchell R, Salkeld E, Chisolm S, Clark M, Jamile M. Path to diagnosis of paroxysmal nocturnal hemoglobinuria: the results of an exploratory study conducted by the Aplastic Anemia and MDS International Foundation and the National Organization for Rare Disorders utilizing an Internet-based survey. SM Clin Med Oncol. 2017;1(1):1-4. Available at: https://www.researchgate.net/publication/331412219 Path to Diagnosis of Paroxysmal_Nocturnal_Hemoglobinuria_ The_Results_of_an_Exploratory_Study_ Conducted by the Aplastic Anemia and MDS International Foundation and the_National_Organization_for_Rare_Di. Accessed December 15, 2020.

4. Shammo J, Mitchell R, Ogborn K, Salkeld E, Chisolm S. Path to diagnosis of paroxysmal nocturnal hemoglobinuria: the results of an exploratory study conducted by the Aplastic Anemia and Myelodysplastic Syndrome International Foundation and the National Organization for Rare Disorders utilizing an internet-based survey, \#3264. Presented at: the 57th Annual Meeting and Exposition of the American Society of Hematology; December 5-8, 2015; Orlando, FL. Available at: https://ash.confex.com/ash/2015/webprogramscheduler/Paper80822.html. Accessed December 15, 2020.

5. Peacock-Young B, Macrae FL, Newton DJ, Hill A, Ariens RAS. The prothrombotic state in paroxysmal nocturnal hemoglobinuria: a multifaceted source. Haematologica. 2018;103(1):9-17.

6. Brodsky RA. How I treat paroxysmal nocturnal hemoglobinuria. Blood. 2009;113(26):6522-27.

7. Parker C, Omine M, Richards S, et al. Diagnosis and management of paroxysmal nocturnal hemoglobinuria. Blood. 2005;106(12):3699-709.

8. Hill A, DeZern AE, Kinoshita T, Brodsky RA. Paroxysmal nocturnal haemoglobinuria. Nat Rev Dis Primers. 2017;3:17028.

9. Weitz I, Meyers G, Lamy T, et al. Cross-sectional validation study of patientreported outcomes in patients with paroxysmal nocturnal haemoglobinuria. Intern Med J. 2013;43(3):298-307.

10. Groth M, Singer S, Niedeggen C, et al. Development of a disease-specific quality of life questionnaire for patients with aplastic anemia and/or paroxysmal nocturnal hemoglobinuria (QLQ-AA/PNH)-report on phases I and II. Ann Hematol. 2017;96(2):171-81.

11. Daly RP, Jalbert JJ, Keith S, Symonds T, Shammo JM. A novel patient reported outcome instrument assessing the symptoms of paroxysmal nocturnal hemoglobinuria. Blood. 2019;134(Suppl 1):385. Available at: https://ashpublications.org/blood/ article/134/Supplement_1/385/426102/ANovel-Patient-Reported-OutcomeInstrument. Accessed December 15, 2020.
12. Niedeggen C, Singer S, Groth M, et al. Design and development of a disease-specific quality of life tool for patients with aplastic anaemia and/or paroxysmal nocturnal haemoglobinuria (QLQ-AA/PNH)-a report on phase III. Ann Hematol. 2019;98(7):1547-59.

13. Schrezenmeier H, Muus P, Socie G, et al. Baseline characteristics and disease burden in patients in the International PNH Registry. Haematologica. 2014;99(5):922-29.

14. Schrezenmeier H, Roth A, Araten DJ, et al. Baseline clinical characteristics and disease burden in patients with paroxysmal nocturnal hemoglobinuria (PNH): updated analysis from the International $\mathrm{PNH}$ Registry. Ann Hematol. 2020;99(7):1505-14.

15. Hinz A, Singer S, Brahler E. European reference values for the quality of life questionnaire EORTC QLQ-C30: results of a German investigation and a summarizing analysis of six European general population normative studies. Acta Oncol. 2014;53(7):958-65.

16. Ueda Y, Obara N, Yonemura Y, et al. Effects of eculizumab treatment on quality of life in patients with paroxysmal nocturnal hemoglobinuria in Japan. Int J Hematol. 2018;107(6):656-65.

17. Cella D, Lai JS, Chang CH, Peterman A, Slavin M. Fatigue in cancer patients compared with fatigue in the general United States population. Cancer. 2002;94(2):528-38. 18. Nishimura J, Yamamoto M, Hayashi S, et al. Genetic variants in $\mathrm{C} 5$ and poor response to eculizumab. N Engl J Med. 2014;370(7):632-39.

19. Badireddy M, Baradhi KM. Chronic anemia. In: StatPearls. StatPearls Publishing; 2020. Available at: https://www.ncbi.nlm. nih.gov/pubmed/30521224. Accessed December 15, 2020.

20. Pacific Heart, Lung, and Blood Institute. Blood disorders: anemia. May 2, 2020. Available at: https://www.phlbi.org/divisions/blood-disorders/anemia/. Accessed December 15, 2020.

21. Mayo Clinic. Anemia. 2020. Available at: https://www.mayoclinic.org/diseasesconditions/anemia/symptoms-causes/syc20351360 . Accessed December 15, 2020.

22. Platzbecker U, Hofbauer LC, Ehninger G, Holig K. The clinical, quality of life, and economic consequences of chronic anemia and transfusion support in patients with myelodysplastic syndromes. Leuk Res. 2012;36(5):525-36.

23. Tomazos I, Sierra JR, Johnston KM, Cheung A, Brodsky RA, Weitz IC. Cost burden of breakthrough hemolysis in patients with paroxysmal nocturnal hemoglobinuria receiving ravulizumab versus eculizumab. Hematology. 2020;25(1):327-34.

24. Oliva EN, Schey C, Hutchings AS. A review of anemia as a cardiovascular risk factor in patients with myelodysplastic syndromes. Am J Blood Res. 2011;1(2):160-66.
25. Cella D, Kallich J, McDermott A, Xu X. The longitudinal relationship of hemoglobin, fatigue and quality of life in anemic cancer patients: results from five randomized clinical trials. Ann Oncol. 2004;15(6):979-86.

26. Berndt E, Kallich J, McDermott A, Xu X, Lee H, Glaspy J. Reductions in anaemia and fatigue are associated with improvements in productivity in cancer patients receiving chemotherapy. Pharmacoeconomics. 2005;23(5):505-14.

27. Efficace F, Gaidano G, Breccia M, et al. Prevalence, severity and correlates of fatigue in newly diagnosed patients with myelodysplastic syndromes. $\mathrm{Br} \mathrm{J}$ Haematol. 2015;168(3):361-70.

28. Harper P, Littlewood T. Anaemia of cancer: impact on patient fatigue and long-term outcome. Oncology. 2005;69(Suppl 2):2-7. 29. Cartenì G, Giannetta L, Ucci G, et al. Correlation between variation in quality of life and change in hemoglobin level after treatment with epoetin alfa 40,000 IU administered once-weekly. Support Care Cancer. 2007;15(9):1057-66.

30. Holzner B, Kemmler G, Greil R, et al. The impact of hemoglobin levels on fatigue and quality of life in cancer patients. Ann Oncol. 2002;13(6):965-73.

31. McKinley C, Richards S, Munir T, et al. Extravascular hemolysis due to C3-loading in patients with PNH treated with eculizumab: defining the clinical syndrome. Blood. 2017;130(Suppl 1):3471. Available at: https://ashpublications.org/blood/ article/130/Supplement\%201/3471/114736/ Extravascular-Hemolysis-Due-to-C3Loading-in. Accessed December 15, 2020. 32. Gao C, Li L, Chen B, et al. Clinical outcomes of transfusion-associated iron overload in patients with refractory chronic anemia. Patient Prefer Adherence. 2014;8:513-17.

33. Takatoku M, Uchiyama T, Okamoto S, et al. Retrospective nationwide survey of Japanese patients with transfusion-dependent MDS and aplastic anemia highlights the negative impact of iron overload on morbidity/mortality. Eur J Haematol. 2013;78(6):487-94.

34. Shreay S, Desrosiers MP, Corey-Lisle P, Payne K. A retrospective study to evaluate the time burden associated with outpatient red blood transfusions indicated for anemia due to concomitantly administered chemotherapy in cancer patients. Support Care Cancer. 2013;21(5):1335-40.

35. Reitan JF, Kudrik FJ, Fox K, van Breda A, Shreay S, Corey-Lisle PK. The burden of blood transfusion: a utilization and economic analysis-a pilot study in patients with chemotherapy-induced anemia (CIA). J Med Econ. 2013;16(5):633-38. 
36. The Joint Commission. Updates to patient blood management certification program. August 5 2020. Available at: https:// www.jointcommission.org/resources/newsand-multimedia/newsletters/newsletters/ joint-commission-online/aug-5-2020/ effective-jan-1-2021-updates-to-patientblood-management-certification-program/. Accessed December 15, 2020.

37. Napolitano LM, Kurek S, Luchette FA, et al. Clinical practice guideline: red blood cell transfusion in adult trauma and critical care. Crit Care Med. 2009;37(12):3124-57.

38. Thakkar RN, Podlasek SJ, Rotello LC, Ness PM, Frank SM. Two-unit red cell transfusions in stable anemic patients.

J Hosp Med. 2017;12(9):747-49.
39. Corey-Lisle PK, Desrosiers MP, Collins H, et al. Transfusions and patient burden in chemotherapy-induced anaemia in France. Ther Adv Med Oncol. 2014;6(4):146-53. 40. Levy AR, Dysart L, Patel Y, et al. Comparison of lost productivity due to eculizumab and ravulizumab treatments for paroxysmal nocturnal hemoglobinuria in France, Germany, Italy, Russia, Spain, the United Kingdom, and the United States. Blood. 2019;134(1):4803. Available at: https:// ashpublications.org/blood/article/134/ Supplement_1/4803/428734/Comparisonof-Lost-Productivity-Due-to-Eculizumab. Accessed December 15, 2020
41. Soliris (eculizumab) prescribing information. Alexion Pharmaceuticals. November 2020. Available at: https://alexion.com/ Documents/Soliris_USPI.pdf. Accessed December 15, 2020.

42. Ultomiris (ravulizumab-cwvz) prescribing information. Alexion Pharmaceuticals. October 2020. Available at: https://alexion. com/Documents/Ultomiris_USPI.pdf. Accessed December 15, 2020.

43. Tenant KF. The FACIT Fatigue Scale (Version 4). 2020. Available at: https://hign. org/consultgeri/try-this-series/facitfatigue-scale-version-4. Accessed December 15, 2020.

\title{
Paroxysmal nocturnal hemoglobinuria: current treatments and unmet needs
}

\author{
Meryem Bektas, PhD, MBA; Catherine Copley-Merriman, MS, MBA; Shahnaz Khan, MPH; \\ Sujata P Sarda, BPharm, PhD; and Jamile M Shammo, MD, FASCP, FACP
}

\section{SUMMARY}

The current standard of care for paroxysmal nocturnal hemoglobinuria (PNH) are the C5 inhibitors eculizumab and ravulizumab, both monoclonal antibodies designed to target the complement protein C5, thereby preventing its cleavage and the formation of the terminal attack complex. C5 inhibitors have yielded substantial improvements in the treatment of $\mathrm{PNH}$ and changed the mortality and morbidity, as well as healthrelated quality of life of patients with the disease. These treatments target underlying intravascular hemolysis; however, they do not address extravascular hemolysis, resulting in incomplete response and remaining symptoms in some patients. Therefore, despite treatment with a C5 inhibitor, some patients still experience anemia with associated fatigue, transfusion needs, and impaired health-related quality of life.

\section{Author affiliations}

Meryem Bektas, PhD, MBA; Catherine Copley-Merriman, MS, MBA; and Shahnaz Khan, MPH, Market Access and Outcomes Strategy, RTI Health Solutions, Research Triangle, NC. Sujata P Sarda, BPharm, PhD, Global Health Economics and Outcomes Research, Apellis Pharmaceuticals, Waltham, MA, and Jamile M Shammo, MD, FASCP, FACP, Department of Internal Medicine, Rush University Medical Center, Chicago, IL.

\section{AUTHOR CORRESPONDENCE: \\ Meryem Bektas, 919.541.6776; \\ mbektas@rti.org}

J Manag Care Spec Pharm 2020;26(12-b):S14-S20

Copyright $\odot 2020$, Academy of Managed Care Pharmacy. All rights reserved. 